\title{
Le rapprochement Chercheur-Consultant en aménagement du territoire : un apport à haute valeur ajoutée?
}

\section{Christine Chemin et Emmanuel Thimonier}

\section{(2) OpenEdition}

10 Journals

\section{Édition électronique}

URL : http://journals.openedition.org/echogeo/11635

DOI : 10.4000/echogeo. 11635

ISSN : 1963-1197

Éditeur

Pôle de recherche pour l'organisation et la diffusion de l'information géographique (CNRS UMR 8586)

\section{Référence électronique}

Christine Chemin et Emmanuel Thimonier, « Le rapprochement Chercheur-Consultant en aménagement du territoire : un apport à haute valeur ajoutée? », EchoGéo [En ligne], Sur le Vif, mis en ligne le 18 février 2010, consulté le 19 avril 2019. URL : http://journals.openedition.org/echogeo/11635 ; DOI : 10.4000/echogeo.11635

Ce document a été généré automatiquement le 19 avril 2019.

\section{c) (7) $९$}

EchoGéo est mis à disposition selon les termes de la licence Creative Commons Attribution - Pas d'Utilisation Commerciale - Pas de Modification 4.0 International 


\title{
Le rapprochement Chercheur- Consultant en aménagement du territoire : un apport à haute valeur ajoutée?
}

\author{
Christine Chemin et Emmanuel Thimonier
}

1 Pour des raisons obscures, une querelle persiste entre le Chercheur et le Consultant, et plus particulièrement sur l'impossible interaction des activités intellectuelles comme la recherche et le conseil. Ces activités répondraient à des logiques distinctes et chacune serait renfermée dans un «monde à part ». Traditionnellement, les sciences sociales ont érigé une séparation entre savoir et action (Vermeulen, 1997). Mais Chercheur et Consultant ne doivent-ils pas répondre à des enjeux communs malgré des divergences établies? La conduite de projets, l'expertise dans le cadre d'études ou d'audits, l'innovation par l'élaboration de nouvelles méthodes de travail ou d'outils d'aide à la décision sont les principaux enjeux d'un cabinet-conseil spécialisé en aménagement. Un laboratoire de recherche en géographie-aménagement n'est-il pas appelé à mener, entre autres, des prestations similaires?

2 Le Chercheur et le Consultant, chacun à leur manière, interviennent selon une posture intellectuelle et une pratique spécifiques. Le Consultant s'appuie davantage sur des faits, tandis que le Chercheur se positionne comme un scientifique aux méthodes académiques basées sur un corpus de connaissances plus théoriques. «A la différence des chercheurs, les consultants produisent des savoirs tournés vers l'action plus que vers la connaissance du réel comme fin en soi " (Simonet, Bouchez, 2003). De même, le Chercheur n'a pas une fonction de conseil à proprement parler : il doit rester dans le cadre d'une démarche de recherche scientifique, c'est-à-dire de progression de la connaissance sur le sujet à traiter, mais « la finalisation de sa recherche le conduit inévitablement à assumer une fonction de conseil et de préconisation" (Nicolas-Lestrat, 2003). Le Chercheur conceptualise davantage sa prestation que le Consultant plus pertinent dans l'accompagnement de son 
commanditaire. Les divergences se trouveraient-elles alors dans les préconisations ou dans les conseils formulés au commanditaire en matière d'aide à la décision?

Une réflexion académique sur l'éventuelle concurrence ou sur la complémentarité entre le Consultant et le Chercheur parait possible. Cependant, il convient auparavant de préciser la fonction du Chercheur à travers sa proximité avec des structures professionnelles en matière d'expertise dans le domaine de l'aménagement.

\section{La science au service de l'expertise}

4 A l'instar d'un bureau d'études, un laboratoire de recherche peut être appelé à réaliser une prestation d'expertise. En effet, le Chercheur peut travailler pour le compte d'une structure extérieure telle qu'une entreprise: c'est le cas de l'Intervenant-chercheur (Henriot, 2005). Ce dernier exerce la fonction de consultant à travers une mission d'expertise en lien avec ses domaines de recherche. Soit il intervient pour le compte de son laboratoire - dans le cadre d'un contrat de recherche -, soit il intervient en son nom propre - pour son propre compte - s'il a créé, parallèlement, une activité libérale... de consultant. Précisons que des disciplines sont plus enclines que d'autres à cette dernière formule: c'est le cas du droit, de l'informatique, ou de la gestion. Ces chercheurs développent une activité de conseil (conseil juridique, solutions informatiques, conseil en stratégie des organisations, etc.). A tel point que, parfois, leurs commanditaires ne savent plus s'ils ont affaire au chercheur, c'est-à-dire au scientifique ou au professeur (entendu comme un "donneur de leçons»), ou au consultant, c'est-à-dire à l'expert (entendu comme un "préconisateur de solutions »). La limite entre les deux fonctions peut donc, dans certains cas, paraitre floue. Mais l'aménagement semble moins concerné par cette double fonction : il est rare qu'un chercheur de cette discipline intervienne en son nom propre en matière d'expertise ; il représente presque toujours son unité de recherche et est toujours identifié comme lui appartenant.

Ainsi, le Chercheur, comme le Consultant, possède un métier de base. En aménagement, il peut être géographe, économiste, gestionnaire, sociologue, juriste, historien, etc.. Définissons différents cadres de cette fonction de chercheur par sa proximité croissante avec les milieux dits professionnels, publics ou privés. Puis interrogeons-nous : cette proximité, longtemps critiquée par la profession, est-elle aujourd'hui devenue évidente en aménagement? N'est-elle pas encore source de réticences, y compris dans les milieux professionnels?

\section{Une plus grande proximité avec les milieux professionnels : l'exemple de l'aménagement}

On l'a vu précédemment, des chercheurs sont plus proches que d'autres, par leur appartenance à certaines disciplines, des milieux professionnels. L'aménagement semble peu à peu se rapprocher de ces milieux, notamment depuis le développement des formations professionnalisantes à l'Université.

7 Dans les années 1980, un premier pas est franchi avec le recrutement, au sein des UFR (Unités de Formation et de Recherche) de géographie-aménagement, de PAST (Professeurs Associés à Service Temporaire) qui partagent leur emploi du temps entre leur activité professionnelle, par exemple de consultant, et des activités d'enseignement 
et de recherche à l'Université. Ceux-ci sont salariés de leur structure professionnelle et dispensent des cours à mi-temps.

Puis, en 2003, la réforme LMD (Licence Master Doctorat) a permis le développement des formations professionnalisantes imposant le recrutement d'un nombre plus important d'enseignants-vacataires, c'est-à-dire de professionnels de l'aménagement dont beaucoup occupent la fonction de consultant.

9 Ces PAST et vacataires apportent un enseignement basé sur des outils, des méthodes et des techniques, enseignements complémentaires à ceux plus théoriques des intervenants académiques. Ils sont très appréciés des étudiants pour leurs apports plus pragmatiques mais aussi pour leurs conseils en matière de recrutement. Ils sont de plus en plus intégrés dans la vie de l'Université en côtoyant régulièrement les enseignants-chercheurs avec lesquels ils unissent leurs compétences pour répondre à des appels d'offres ou pour participer à des programmes de recherche.

10 Le recrutement plus important de ce type d'enseignants établit donc un premier type de rapprochement entre le monde académique et le monde professionnel. Effectivement, ce rapprochement est nécessaire pour l'Enseignant-chercheur en aménagement : ce type de contact lui permet de créer, de développer et de vérifier les théories sur lesquelles il s'appuie dans la préparation de ses cours, évitant ainsi un trop fort décalage entre l'avancée de la recherche et les pratiques de terrain. Il lui permet également une révision permanente du contenu de ses enseignements mais aussi une refonte constante des processus de production de connaissances scientifiques (Paturel, Voyant, 2004).

11 Autre signe évocateur de rapprochement Consultant - Chercheur dans le domaine de l'aménagement: les agences d'urbanisme sont devenues des employeurs de plus en plus fréquents pour des aménageurs ayant un profil de chercheur. Une nouvelle fonction est d'ailleurs apparue au sein de ces structures. A celle de chargé de missions ou de chargé d'études s'ajoute désormais la fonction de chargé de recherche ; à la première correspond un recrutement de niveau master ou ingénieur $(\mathrm{Bac}+5)$, tandis qu'à la seconde correspond un recrutement de niveau doctorat $(\mathrm{Bac}+8)$. Les méthodes de travail d'un chargé de recherche correspondent à celles d'un chercheur et donnent des résultats convaincants.

Le Chercheur en aménagement peut également exercer ses compétences scientifiques directement au sein d'une entreprise ou d'une collectivité : en effet, il aura pu être recruté par le biais d'une première mission de recherche appliquée via une thèse en CIFRE (Convention Industrielle de Formation par la REcherche). Cette formule consiste en l'embauche, par exemple par un cabinet-conseil, d'un jeune diplômé de niveau Bac +5 (ingénieur ou universitaire) en vue de la réalisation d'une thèse de doctorat. Cette convention est tripartite: elle est signée entre le doctorant, le cabinet-conseil et le laboratoire de recherche de rattachement du doctorant. La mission de ce dernier, consultant salarié du cabinet dans cet exemple, repose sur le caractère innovateur de son travail. Ce type de partenariat a déjà séduit quelques bureaux d'études spécialisés en aménagement qui voient en ces chercheurs des collaborateurs capables d'apporter des solutions originales à des problématiques souvent complexes. Ces solutions correspondent, la plupart du temps, à la mise en place de méthodes de travail innovantes permettant de développer l'activité du cabinet qui peut alors s'ouvrir sur de nouveaux champs d'interventions (annexes 1 et 2). 

connaissances et des compétences au service de l'innovation. Pour les trois partenaires, plusieurs enjeux sont à noter : pour le cabinet, il s'agit de répondre à une stratégie de recherche et développement; pour le doctorant, de lui apporter une formation professionnalisante; pour le laboratoire, de travailler sur une thématique ouverte sur le monde professionnel; pour le laboratoire et pour le cabinet, de passer un contrat de collaboration.

Le doctorant est donc au centre du système : il constitue le relais entre le laboratoire et le cabinet. Il apporte, à ce dernier, des modes de pensée différents, des réflexes, des connaissances permettant une meilleure qualité de service auprès de ses clients. Le doctorant apporte au laboratoire une connaissance et une expertise de terrain, un mode de fonctionnement, des compétences techniques pouvant faire l'objet de cours au sein de formations professionnalisantes de l'Université.

Si ce rapprochement des milieux professionnels paraît aujourd'hui nécessaire pour le Chercheur en aménagement, celui-ci a pu faire l'objet de vives critiques, tant par ses pairs que par le Consultant qui, aujourd'hui encore, le perçoit mal dans sa fonction. Par ses pairs, le Chercheur a pu être considéré comme un serviteur complice et intéressé de l'Etat, délaissant quelque peu la science au profit d'une activité plus pratique ou factuelle peu valorisante pour l'Académie. Pour le Consultant, le Chercheur est encore perçu, aujourd'hui, comme trop conceptuel ou trop théorique dans son travail laissant présager, notamment, une certaine inadaptabilité de ses préconisations à la problématique et, plus généralement, au terrain d'étude.

\section{Le Chercheur en aménagement critiqué et parfois mal perçu}

Le Chercheur essuie régulièrement des critiques quant à sa fonction voire à son utilité. Cette fonction de «savant » reste aujourd'hui considérée comme floue ou opaque. Que cherche-t-il ? Quels sont les conclusions ou les résultats de ses travaux ? A qui profitentils?

17 Par le passé, le Chercheur en aménagement n'a pas échappé à la règle : on l'accusait de complicité avec les plus hautes sphères de l'Etat. Dans son ouvrage intitulé Expertise et aménagement du territoire. L'Etat savant (1996), G. Massardier, chercheur en science politique, s'intéresse aux relations entretenues, tout au long du XX⿳亠丷⿵冂丶 ${ }^{2}$ siècle, entre les savants en sciences sociales - les urbanistes et les géographes - et «l'administratif (Ministère de la Reconstruction et de l'Urbanisme, DATAR (Délégation à l'Aménagement du Territoire et à l'Action Régionale), grands corps de l'Etat...) ». Il résume son ouvrage en expliquant que son étude passe au crible la stratégie très intéressée de ces urbanistes et géographes :

«Qu'il s'agisse des « urbanistes les plus autorisés » des années 1930-1950, ou des «nouveaux géographes» des années 1970-1980, on retrouve des pratiques identiques : la construction d'un groupe d'experts, au départ « hérétique » dans sa discipline, qui multiplie les positions de pouvoir dans et hors de l'espace des savants (administration, politique, consultanat). Entre savoir et pouvoir : tel semble être le statut de l'expertise savante. Empiriquement, il est montré que la dichotomie wéberienne entre les "vocations" savante et administrative peut laisser place à une "complicité » basée sur un système producteur de savoir «théorique » autonome ». 

décentralisation et permettant aux collectivités un recours quasi systématique aux consultants avant leur prise de décisions, ne permet-elle pas de reprendre cette critique de la complicité entre l'acteur-décideur et l'acteur-conseiller ? Le Consultant n'est-il pas devenu un interlocuteur privilégié voire complice de certaines collectivités tentées de faire confiance $\mathrm{au}(\mathrm{x})$ même(s) prestataire(s), y compris dans un contexte législatif incitant de plus en plus à l'appel d'offres? Effectivement, il n'est pas rare, en France, de retrouver systématiquement les mêmes cabinets-conseil dans tous les projets d'aménagement d'envergure ; laissant penser un manque de transparence dans la plupart des appels d'offres. Deux raisons peuvent être avancées: d'une part, ces cabinets, qui emploient plusieurs dizaines de salariés par le biais de leurs filiales et de leurs établissements implantés sur l'ensemble du territoire français, sont annoncés comme concurrents ; en fait, ils entretiennent de véritables liens de complicité en se partageant les projets les plus remarquables et les plus rentables, laissant les autres aux cabinets de moindre notoriété. Les premiers quadrillent ainsi de vastes territoires imprenables par les seconds sauf, éventuellement, à s'associer avec eux. D'autre part, la signature reconnue de ces cabinets notoires est souvent perçue comme un gage de sérieux et de qualité par leurs commanditaires publics compte tenu des tarifs de leurs prestations et de la présence, en leur sein, de consultants considérés comme "spécialistes " voire « experts » des questions traitées. Toutefois, ces commanditaires sont aujourd'hui moins dupes, faisant plus souvent appel à des cabinets moins célèbres mais tout aussi consciencieux et efficaces.

uoi qu'il en soit, en règle générale, les cabinets-conseil restent encore très réticents à l'embauche d'un collaborateur au profil de chercheur, craignant des prestations trop académiques et des résultats peu applicables pour leurs clients. Certes, les thèses en CIFRE évoquées plus haut constituent une stratégie d'embauche d'un ConsultantChercheur intéressante, mais cette pratique reste limitée. Cette critique du Chercheur est encore prégnante dans un monde où la recherche reste, finalement, très méconnue. D'autant que, pour certains cabinets, le recrutement d'un tel collaborateur peut signifier une plus forte rémunération. C'est là un point qui marque encore une distance.

Le positionnement du Chercheur dans le jeu des acteurs de l'expertise n'est pas toujours clair. Cependant, les critiques dont il peut faire l'objet ne doivent pas occulter une certaine prise de conscience. En effet, l'association entre le Chercheur et le Consultant tend à se développer dans ce domaine et donnent des résultats très intéressants. Et ce rapprochement est aujourd'hui de plus en plus souhaité par les services de l'Etat et les collectivités, conscients qu'un tel partenariat ne peut que bénéficier à leur prise de décisions sans que le coût supporté n'en soit forcément plus onéreux.

\section{Chercheur et Consultant : des divergences sources de complémentarité, de rapprochement et d'enrichissement mutuel}

21 Les trois grandes fonctions du Chercheur - objectif d'avancement de l'état de la connaissance et des savoirs, innovation dans les méthodes et dans les outils, apport d'expertise à une structure extérieure - n'est pas sans rappeler trois des grandes fonctions du Consultant: la veille quotidienne d'informations pour améliorer sa 
réactivité, l'innovation dans ses méthodes et dans ses outils de travail, l'apport d'une expertise. Cette assertion tendrait à évoquer une certaine concurrence, sur le fond, entre le Consultant et le Chercheur. Mais, en fait, ce sont des divergences d'approches et de méthodes de travail entre le Chercheur et le Consultant qui les rendent complémentaires. Cette complémentarité rapproche aujourd'hui ces deux acteurs incontournables de l'aménagement et les enrichit mutuellement.

\section{Des divergences notables entre Chercheur et Consultant...}

M. Kubr (Bureau International du Travail, 1993) établit plusieurs facteurs de divergences entre les activités de recherche et les activités de conseil. Les enseignements de cette analyse, réalisée à partir du conseil en management des organisations, ont été repris ciaprès car ils s'appliquent tout aussi bien à l'aménagement.

Le premier enseignement réside dans le degré de liberté d'action plus important côté Chercheur que côté Consultant: tandis que le premier définit la problématique sur laquelle il souhaite travailler, le second doit suivre celle imposée par son client ou son commanditaire même s'il peut participer à sa définition. Le Consultant doit respecter le délai imparti de la commande et donc bien organiser son temps de travail alors que le second n'a pas forcément d'échéance (sauf quand il répond à une commande d'expertise). Enfin, le Chercheur peut orienter et faire varier l'évolution de son travail selon ses prérogatives de recherche et celles de son laboratoire alors que le Consultant ne dispose que d'une marge de manœuvre très limitée en termes de décision, souvent très cadrée par la mission.

Le deuxième enseignement réside dans l'objectif des travaux réalisés : pour le Chercheur, il s'agit de faire avancer la connaissance et les savoirs, via une grande rigueur scientifique et, au final, de se risquer à l'élaboration de théories. Pour le Consultant, il s'agit de répondre à une question permettant un apport de solution sans forcément prendre appui sur une rigueur de type scientifique. Les travaux réalisés par le Chercheur sont généralement publiés tandis que ceux du Consultant ne sont que très rarement diffusés. La confidentialité est de rigueur dans le monde du conseil tandis que dans celui de la science, la publication, notamment à l'international, constitue une obligation.

Le troisième enseignement réside dans l'évaluation des travaux: pour le scientifique, c'est la confrontation et la soutenance de ses travaux face à ses pairs qui en cautionnent la qualité et le niveau. Pour le Consultant, c'est le commanditaire, c'est-à-dire l'utilisateur, qui est en mesure de l'évaluer puisqu'il va s'appuyer sur les préconisations émises pour sa prise de décisions.

La simple lecture de cette liste - non exhaustive - des divergences entre le Chercheur et le Consultant semble les opposer. Mais, finalement, ces divergences d'approches et de méthodes de travail ne constituent-elles pas, justement, la raison qui les incite à se rapprocher et à s'enrichir mutuellement ? Ne les rendent-ils donc pas complémentaires ? $\mathrm{Au}$ final, leurs commanditaires ne sont-ils pas les véritables bénéficiaires de ce rapprochement? 


\section{... à la base d'un enrichissement respectif}

Effectivement, malgré des divergences persistantes entre ces deux acteurs, l'heure est au rapprochement et à la complémentarité, notamment en sciences de gestion mais aussi en aménagement du territoire.

P. Cabin (in Simonet, Bouchez, 2003) avance l'idée selon laquelle le Consultant peut jouer le rôle de relais entre la théorie et la pratique, c'est-à-dire d'intermédiaire entre le monde de la Recherche et le monde de l'Entreprise. Selon lui, les entreprises, quand elles font appel à un prestataire extérieur pour traiter des problèmes relevant des sciences humaines, s'adressent dans $80 \%$ des cas à des cabinets-conseil, dans $13 \%$ des cas à des experts indépendants et dans $7 \%$ des cas à des équipes de recherche. Comme le Consultant vend des outils opérationnels et parle le langage de l'Entreprise, il accommode les théories scientifiques en en choisissant les aspects les plus performants, en les reformulant dans un langage et une grammaire clairs et accessibles pour leurs commanditaires.

9 Cette analyse un peu sévère pour le Consultant, qui ne serait qu'un interprète sélectif des travaux du Chercheur, apporte un premier élément tendant à prouver une certaine complémentarité entre ces deux acteurs.

Au-delà de son analyse sur les divergences entre le Chercheur et le Consultant, M. Kubr évoque la complémentarité entre le Chercheur et le Consultant dans le domaine des sciences de gestion. Il constate une observation, une veille réciproque (une surveillance) et un phénomène d'allers-retours entre les deux acteurs leur permettant de mieux se connaître et de mieux se comprendre. Cette connaissance et cette compréhension accrues peuvent être à la base de partenariats dans lesquels les deux partis sont gagnants: gagnants en termes de temps (en gains de productivité) par un échange de méthodes ou d'informations, gagnants par un développement ou une évolution d'activité, gagnants en termes d'image puis de notoriété (par la diffusion de résultats de recherche par le biais de missions d'expertise), et gagnants en termes d'apprentissages méthodologiques. Les missions de recherche-action en gestion constituent des exemples très éloquents.

Les éléments qui suivent comparant le Consultant et le Chercheur sont issus d'une proposition de collaboration en réponse à un appel d'offres (2004). Cette proposition a été rédigée conjointement entre le cabinet-conseil M\&P - La belle idée et la Faculté de sciences économiques, de gestion et d'informatique de l'Université Lumière (Lyon II). La prestation portait sur la mise en valeur des activités de recherche en micro et en macro-économie, en gestion stratégique des organisations, et en informatique auprès des entreprises. L'argumentation de la proposition, mettant en avant le partenariat laboratoire de recherche - cabinet-conseil, précisait :

«- le Consultant doit susciter l'interrogation puis se positionner en accompagnant le recadrage de la réflexion du ou des commanditaires d'une mission; il travaille sur les enjeux perçus ou non perçus par ceux-ci et en accompagne la mise en œuvre ;

- le Chercheur alimente la réflexion, les questionnements ; il apporte un recul sur les outils d'analyse et de réponse disponibles sur le marché et met en perspective les enjeux. Il s'inscrit à la fois dans l'histoire et l'actualité ; il évite la « course au temps » et, par son travail d'analyse et de propositions, alimente le consultant en informations et données nouvelles ». 

relation basée sur un processus d'allers-retours permanents entre les deux, c'est-à-dire entre la science et le conseil, l'un l'autre s'alimentant? Les travaux du Chercheur constituent une matière très précieuse pour ceux du Consultant, notamment en matière d'innovation (concepts, modèles), corroborant les nouveaux outils et les nouvelles méthodes issus de l'expérience de terrain de celui-ci. Comme le précise F. Vermeulen 
(1997), «à défaut d'être utile, le savoir est stérile, et seule la confrontation par la pratique permet d'éprouver la validité d'une théorie ».

Mais la collaboration entre le Consultant et le Chercheur n'est pas encore chose fréquente en aménagement. Toutefois, le recrutement dans ce domaine montre un réel rapprochement entre le Chercheur et le Consultant, les deux pouvant parfois constituer une seule et même personne dans le cadre d'enseignements associés ou d'une thèse professionnelle.

En matière de recherche-action ou de travaux d'expertises, le modèle anglo-saxon des sciences de gestion semble avoir montré la voie au cours de ces dernières années. En d'autres termes, les sciences de gestion paraissent avoir prouvé aux disciplines telles que l'aménagement du territoire qu'un partenariat Chercheur-Consultant était bénéfique voire incontournable. Les travaux menés en collaboration entre ces deux types d'acteurs donnent des résultats probants en termes d'enrichissement respectif mais aussi en termes de conseil et d'innovation auprès des commanditaires d'expertises. Ces derniers, qu'ils soient publics ou privés, sont d'ailleurs de plus en plus intéressés par ce type de partenariats qui, selon eux, apportent une réelle valeur ajoutée aux prestations.

In urban planning new partnership practices seem to appear through the realization of expertises (studies, projects engineering, audits...). Although the appeal to consulting firms - to consultants - and in the scientific laboratories - to researchers - by the State services or local government, exists for a long time now, the partnership between consultants and researchers is recent. Even though this partnership is not systematic in urban planning, it brings a contribution with a high added value in the services, according to the public partners.

\section{BIBLIOGRAPHIE}

Ardoino J., 1989. D'une ambiguïté propre à la recherche-action aux confusions entretenues par les pratiques d'intervention. Pratiques de Formation/Analyses, $\mathrm{n}^{\circ} 18$ (disponible sur http://wwwufr8.univ-paris8.fr/pfa/18presentation.html). Paris, 7 p.

De Brabandère L., 2004. Le management des idées. De la créativité à l'innovation (2 ${ }^{\mathrm{ème}}$ édition). Paris, Dunod, $244 \mathrm{p}$.

Dumoulin L., La Branche S., Robert C., Warin P., 2005. Le recours aux experts. Raisons et usages politiques. Grenoble, Presses Universitaires de Grenoble (PUG), 488 p.

Grimand A., Laurent P., Martinet A.-C., Tannery F., Wissler M., 1999. Les relations entreprises / services de conseils: apprentissage et dynamiques territoriales. Lyon, EURISTIK (UPRES-A CNRS 5055), $54 \mathrm{p}$.

Henriot J., 2005. Génération et gestion d'une dynamique coopérative entre intervenantschercheurs et acteurs praticiens. Cas d'expérimentation en sciences de gestion. Thèse de doctorat, volume 1, Université de Lyon Jean-Moulin (Lyon III), Lyon, 354 p. 
Henry O., 1992. Entre savoir et pouvoir. Les professionnels de l'expertise et du conseil. Actes de la recherche en sciences sociales.Paris, $n^{\circ} 95$, Maison des Sciences de l'Homme, Ed. de Minuit, 18 p.

Institut Socio-Economique des ORganisations (ISEOR), 1992. Qualité du conseil et mutation du secteur public : professionnalisme des consultants. Actes du $4{ }^{\text {ème }}$ colloque. Paris, Economica, 357 p.

Institut Socio-Economique des ORganisations (ISEOR), 1999. Les conseils aux entreprises : professionnalisme des consultants. Actes du $11^{\mathrm{ème}}$ colloque. Paris, Economica, $251 \mathrm{p}$.

Institut Socio-Economique des ORganisations (ISEOR), Institut d'Administration des Entreprises (IAE) Université de Lyon Jean-Moulin (Lyon III), 2004. First International Co-Sponsored Conference Research Methods Division Academy of Management (USA). Traversée des frontières entre méthodes de recherche qualitatives et quantitatives, volume 1 édité par Savall H., Bonnet M., Péron M.. Lyon, 770 p.

Jacob S., Génard J.-L., 2004. Expertise publique. Bruxelles, Editions de l'Université de Bruxelles, 165 p.

Kubr M., 1993. Le conseil en management. Guide pour la profession (2 ${ }^{\mathrm{ème}}$ édition révisée). Genève, BIT Genève, 662 p.

Massardier G., 1996. Expertise et aménagement du territoire. L'Etat savant. Paris, L'Harmattan, $286 \mathrm{p}$.

Mreiben A., 2000. Le conseil en management. Paris, Village Mondial, $192 \mathrm{p}$.

Müller P., 2000. L'analyse cognitive des politiques publiques : vers une sociologie politique de l'action publique. Revue française de science politique, volume 50, $\mathrm{n}^{\circ} 2$. Paris, Presses Universitaires de France (PUF), 18 p.

Nicolas Y., 1994. L'expert et l'universitaire : la fin d'une guerre froide ? Sciences de la société (Les Cahiers du LERASS), $\mathrm{n}^{\circ} 32$. Toulouse, $14 \mathrm{p}$.

Nicolas-Lestrat P., 2003. La relation de consultance. Une sociologie des activités d'étude et de conseil. Paris, L'Harmattan, $252 \mathrm{p}$.

Restier-Melleray C., 2000. Experts et expertise scientifique, le cas de la France. Revue française de science politique, $\mathrm{n}^{\circ} \mathrm{IV}$. Paris, Presse Universitaire de France (PUF), $39 \mathrm{p}$.

Simonet J., Bouchez J.-P., Pelade J., Gilbert P., 2003. Le conseil. Le livre du consultant et du client. Paris, Editions d'Organisation, $553 \mathrm{p}$.

Stern P., Tutoy P., 2001. Le métier de consultant. Principes, méthodes, outils (3 ${ }^{\text {ème }}$ édition). Paris, Editions d'Organisation, $288 \mathrm{p}$.

Thimonier E., 2005. L'efficacité du découpage territorial : contribution méthodologique pour déterminer des territoires d'expertise adaptés de la Métropole lyonnaise. Deuxième rapport sur l'état d'avancement à l'ANRT (thèse CIFRE), Université de Lyon Jean-Moulin (Lyon III), M\&P - La belle idée, Lyon, $45 \mathrm{p}$.

Thimonier E., 2006. Aide à la décision : innovation, transfert, dynamique territoriale. Limas, étude pour le compte du CREUSET, 2 volumes, $55 \mathrm{p}$.

Thimonier E., 2009. L'efficacité du découpage territorial : contribution méthodologique pour déterminer des territoires d'expertise adaptés de la Métropole lyonnaise. Thèse de doctorat (en CIFRE), Université de Lyon Jean-Moulin (Lyon III), Lyon, 400 p.

Trepos J.-Y., 1996. La sociologie de l'expertise. Paris, Que sais-je ? n³119, Presses Universitaires de France (PUF), $127 \mathrm{p}$. 
Vermeulen F., 1997. Chercheurs, consultants : le dialogue impossible? Les Petits Déjeuners "Confidences ». Ecole de Paris du Management. Paris, séance du 18 mars 1997, 11 p.

Villette M., 2003. Sociologie du conseil en management. Paris, La Découverte, 128 p.

Weber M., 2004. Le savant et le politique : une nouvelle traduction. Edition traduite par ColliotThélène C.. Paris, La Découverte, 206 p.

ANNEXES 
L'adaptabilité des territoires d'expertise constitue un enjeu fort pour les acteurs de l'aménagement. En effet, pour mener une action, ils choisissent le découpage existant qui leur paraît constituer le meilleur compromis pour la réalisation de leur étude. La principale limite de ces découpages est leur généralisation en constituant la base territoriale d'un grand nombre de problématiques d'études. Mais, par exemple, le territoire d'expertise adapté pour la thématique de l'immobilier de bureaux est-il le même que celui des déplacements de personnes? La réponse est non : a priori, le premier se limite à des îlots urbains de quelques $\mathrm{km}^{2}$ bien localisés dans la ville, tandis que le second s'organise en réseaux reliant des pôles à une échelle quasi-régionale. Pourtant, si un donneur d'ordres commande une expertise sur ces deux thématiques, il demandera à ce que ces thématiques soient abordées selon son territoire de compétences. S'il y a plusieurs commanditaires, le territoire retenu pourra être un zonage INSEE ou le territoire de l'acteur couvrant la maille la plus large. Bref, un territoire prédéfini, de compromis, et souvent inadapté aux thématiques en question.

Toutefois, de plus en plus, ces commanditaires d'expertises prennent conscience de cette aberration et autorisent parfois leurs prestataires à ne pas respecter les périmètres des territoires imposés. Ce fut le cas, en 2002, lorsque le SGAR (Secrétariat Général aux Affaires Régionales) de la Préfecture de région Rhône-Alpes a commandé au cabinet M\&P La belle idée une série d'audits portant sur dix pôles de compétences en région lyonnaise. Le territoire d'expertise initial était celui de la Directive Territoriale d'Aménagement de l'aire métropolitaine lyonnaise. Celle-ci englobait des territoires sans rapport avec toutes les thématiques à traiter, tandis que d'autres territoires, proches des limites de celle-ci et adaptés à la réflexion, étaient ignorés. Le SGAR a alors autorisé le cabinet à déterminer des territoires plus appropriés à chaque pôle.

Au-delà de cet exemple, et pour chaque prestation de ce type, ce cabinet n'hésitait pas à proposer des découpages territoriaux qui lui paraissaient plus adaptés au regard de son expérience et de sa connaissance de terrain. Mais ces découpages proposés demeuraient perfectibles et le besoin d'un outil méthodologique permettant de définir des périmètres d'expertise propres à chaque thématique s'est avéré nécessaire.

Lorsque la décision fut prise de mettre au point un tel outil, ce cabinet a souhaité le cautionner scientifiquement pour une meilleure reconnaissance auprès de ses clients. Pour ce faire, cette réflexion devait être conduite dans le cadre d'un travail académique, mais aussi factuel, soit un travail alliant recherche fondamentale et recherche appliquée. La formule de la thèse en CIFRE a été retenue. Le doctorant en charge de ce travail était aussi consultant de ce cabinet.

$\mathrm{Au}$ final, outre l'apport scientifique, cette thèse professionnelle a permis la mise au point d'un outil d'aide à la décision pour des collectivités ou des services de l'Etat, acteurs confrontés à cette problématique de territoires à géométrie et à temporalité variables.

Source : Thimonier E., 2005. L'efficacité du découpage territorial : contribution méthodologique pour déterminer des territoires d'expertise adaptés de la Métropole lyonnaise. Deuxième rapport sur l'état d'avancement à I'ANRT (thèse CIFRE), Université de Lyon Jean-Moulin (Lyon III), M\&P - La belle idée, Lyon, $45 \mathrm{p}$. 


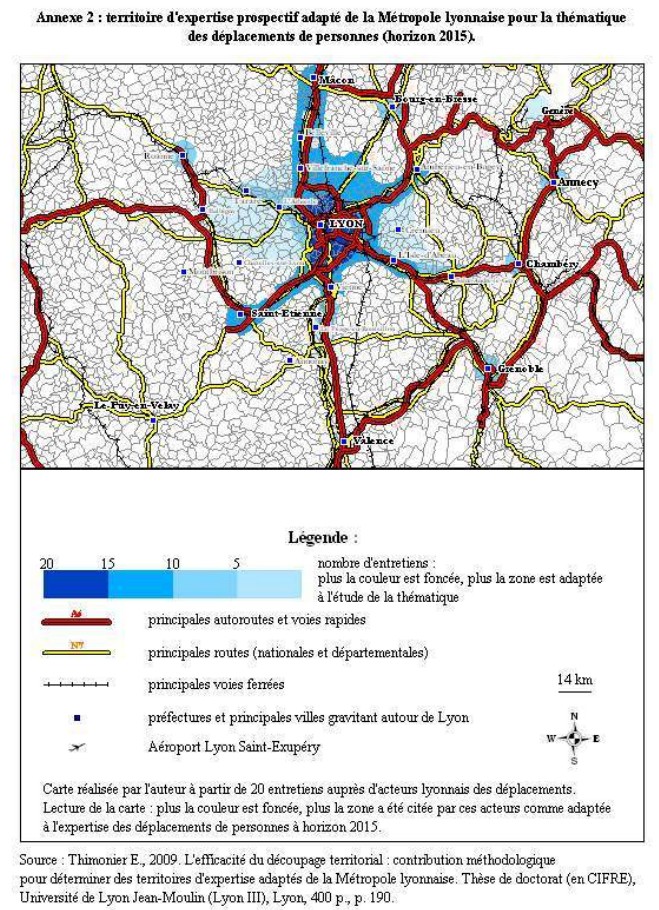

\section{RÉSUMÉS}

En aménagement du territoire, de nouvelles pratiques partenariales semblent émerger lors de la réalisation d'expertises (études, ingénierie de projets, audits, etc.). Si le recours aux cabinetsconseils - au Consultant - et aux laboratoires scientifiques - au Chercheur - par les services de l'Etat ou les collectivités territoriales existe depuis de nombreuses années, le rapprochement et le partenariat entre les consultants et les chercheurs est récent. Certes, cette pratique n'est pas encore systématique en aménagement du territoire mais elle constitue, selon les commanditaires publics, un apport à haute valeur ajoutée dans les prestations de services.

In urban planning new partnership practices seem to appear through the realization of expertises (studies, projects engineering, audits...). Although the appeal to consulting firms - to consultants - and in the scientific laboratories - to researchers - by the State services or local government, exists for a long time now, the partnership between consultants and researchers is recent. Even though this partnership is not systematic in urban planning, it brings a contribution with a high added value in the services, according to the public partners.

\section{INDEX}

Mots-clés : chercheur, consultant, divergences, partenariat, aménagement du territoire, expertise

Keywords : researcher, consultant, divergence, partnership, urban planning, expertise 


\section{AUTEURS}

\section{CHRISTINE CHEMIN}

Christine Chemin est doctorante à l'Université de Lyon Jean-Moulin (Lyon III) et membre de l'UMR 5600 Environnement, Ville, Société, Centre de Recherche en Géographie et Aménagement (CRGA). Ses dernières publications : Belmessous F., Chemin C., Chignier-Riboulon F., Commerçon N., Trigueiro M., Zeph M., 2005. Large housing estates in France : Opinions and prospects of inhabitants, Utrecht, Urban and Regional research centre Utrecht, Utrecht University, 73 p. Belmessous F., Chemin C., Chignier-Riboulon F., Commerçon N., Trigueiro M., Zeph M., 2004. Large housing estates in France : policies and practices, Utrecht : Urban and Regional research centre Utrecht, Utrecht University, $57 \mathrm{p}$.

christine-chemin@orange.fr

\section{EMMANUEL THIMONIER}

Emmanuel Thimonier est ATER à l'Université de Lyon Jean-Moulin (Lyon III) et membre de l'UMR 5600 Environnement, Ville, Société, Centre de Recherche en Géographie et Aménagement (CRGA) Ses dernières publications : Thimonier E., 2009. Quand l'Université façonne la Ville... une relation de longue haleine. Lyon : un atlas des dynamiques universitaires. Traits d'Agences (supplément à Traits Urbains), Fédération Nationale des Agences d'Urbanisme (FNAU), n³2, été 2009, 1 p., p. 13. Mochon J., Thimonier E., 2008. L'apprentissage est en pleine mutation et c'est une chance pour les entreprises. CGA Informations, $\mathrm{n}^{\circ} 110,4$ p., pp. 4-5 et pp. 10-11.

emmanuel.thimonier@orange.fr 